\title{
Anestesia total intravenosa libre de opioides en un paciente con síndrome de Joubert: caso clínico
}

Opioid free total intravenous anesthesia in Joubert syndrome: Case report

Ezequiel L. Fernández MD. ${ }^{1}$, Gonzalo M. Jacquez MD. ${ }^{1}$

\begin{abstract}
Joubert syndrome (JS) is a rare autosomal recessive disorder characterized by abnormal eye movements, respiratory pattern abnormalities, anatomical airway alterations, mental retardation and hypoplasia/aplasia of the cerebellar vermis confirmed by magnetic resonance imaging. This case report describes the successful management of a patient with JS operated of cholesteatoma under $100 \%$ opioid-free total intravenous general anaesthesia. We also provide a brief review of JS, its anaesthetic implications and opioid-free anaesthesia (OFA) technique.
\end{abstract}

\section{RESUMEN}

El síndrome de Joubert (SJ) es una enfermedad autosómica recesiva poco frecuente caracterizada por trastornos oculares, respiratorios, alteraciones anatómicas de la vía aérea, retraso mental e hipoplasia/aplasia del vermis cerebeloso constatada mediante resonancia magnética. Presentamos un caso exitoso de paciente con SJ operado de colesteatoma bajo anestesia general endovenosa total $100 \%$ libre de opioides. Asimismo, realizamos una breve revisión del SJ, sus implicaciones anestésicas y de la técnica de anestesia libre de opioides.

\section{Key words:}

Síndrome de Joubert, total intravenous anesthesia, dexmedetomidina

\section{Palabras clave:}

Joubert syndrome, total intravenous anesthesia, dexmedetomidine

Centro de Educación Médica e Investigaciones Clínicas "Norberto Quirno" (CEMIC)

Fecha de recepción: 02 de junio de 2019

Fecha de aceptación: 19 de agosto de 2019

\section{ORCID}

https://orcid.org/0000-0002-6470-677X

Corespondencia:

Ezequiel L. Fernández

el_fernandez22@hotmail.com 


\section{Introducción}

E I síndrome de Joubert es una enfermedad autosómica recesiva poco frecuente, descripta por primera vez en 1969[1] y con una prevalencia estimada de 1/100.000.

Sus principales manifestaciones clínicas son apraxia oculomotora, retardo mental en diferentes grados, hipotonía y alteraciones del ritmo respiratorio con un patrón de apneas/hiperpneas. El diagnóstico de certeza es imagenológico: constatando la presencia de agenesia total o parcial del vermis cerebeloso.

Debido a que tanto los opioides como los anestésicos volátiles pueden potenciar las alteraciones respiratorias aumentando el riesgo de aparición de apneas potencialmente mortales en el POP[1]-[8], la mayoría de los reportes de anestesia en estos pacientes son de anestesia regional, sedaciones o esquemas de anestesia general que intentan evitarlos, pero siempre incluyen algún tipo de opioide, anestésico volátil o relajante muscular.

Presentamos lo que, según la bibliografía publicada, consideramos ser el primer caso reportado de un paciente con síndrome de Joubert operado de colesteatoma bajo anestesia general total endovenosa (TIVA) 100\% libre de opioides, sin relajación neuromuscular.

\section{Caso clínico}

Paciente de 12 años $(50 \mathrm{~kg}$ ) se presenta en nuestra institución para una mastoidectomía y timpano- plastia con monitoreo de nervio facial bajo anestesia general.

Durante la evaluación preanestésica la paciente se presenta lúcida, tensión arterial (TA) 120/72 mmHg, frecuencia cardíaca (FC) 80 lpm y una saturación de 99\% respirando aire ambiente, evaluado con oxímetro de pulso. Alteraciones anatómicas de la estructura facial, paladar ojival, úvula bífida, Mallampati I, trastorno de los movimientos oculares y retraso madurativo. Sus padres no refieren alteraciones de los patrones respiratorios ni apneas.

Luego de obtener el consentimiento informado de los padres, la paciente ingresa a quirófano, acompañada por su madre, cumpliendo 8 horas de ayuno. Se le realiza monitoreo estándar (oximetría de pulso, electrocardiografía, dióxido de carbono espirado y presión arterial no invasiva). Después de colocar el acceso venoso se administra una dosis de carga de dexmedetomidina $1 \mathrm{mcg} / \mathrm{kg}$, ketamina 0,50 mg/kg y lidocaína $3 \mathrm{mg} / \mathrm{kg}$.

La inducción se realiza con propofol $2 \mathrm{mg} / \mathrm{kg}$, sulfato de magnesio $40 \mathrm{mg} / \mathrm{kg}$ y Midazolam 0,06 mg/kg.

No se utilizó ningún tipo de relajante muscular con el objetivo de permitir la monitorización del nervio facial en el intraoperatorio y minimizar cualquier tipo de alteración en la ventilación durante el postoperatorio. La intubación se realiza sin complicaciones, se utilizó una rama curva articulada $\mathrm{N}^{\circ} 3$ y un tubo $6,5 \mathrm{~mm}$ de diámetro interno. El mantenimiento se realizó mediante anestesia total intravenosa con una infusión de propofol $6 \mathrm{mg} / \mathrm{kg} / \mathrm{h}$, ketamina $0,25 \mathrm{mg} /$ $\mathrm{kg} / \mathrm{h}$, dexmedetomidina $0,5 \mathrm{mcg} / \mathrm{kg} / \mathrm{h}$ y lidocaina 1,5 $\mathrm{mg} / \mathrm{kg} / \mathrm{h}$ (Figura 1).

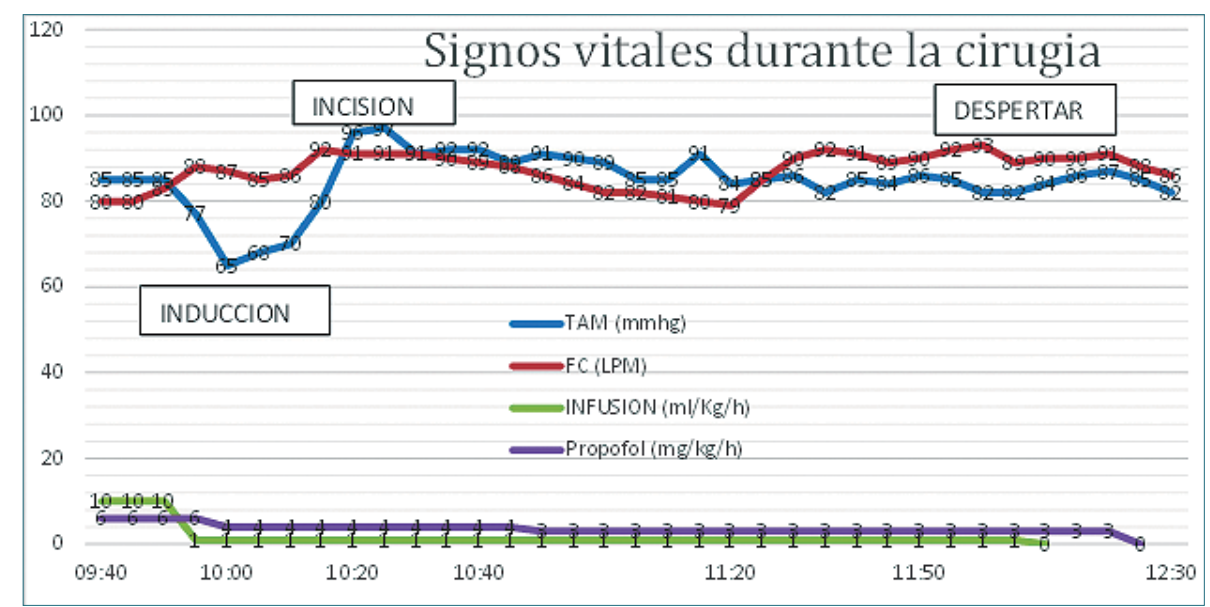

Figura 1. La figura muestra los signos vitales de nuestro paciente junto con las dosis utilizadas en la infusión de las drogas anestésicas durante la cirugía. Tensión arterial media (TAM). Frecuencia cardíaca (FC). 
Previo a la incisión se administró dexamentasona $0,2 \mathrm{mg} / \mathrm{kg}$, ketorolac $1 \mathrm{mg} / \mathrm{kg}$ y una carga de $500 \mathrm{mg}$ de paracetamol para potenciar el manejo multimodal de la analgesia. Luego de realizar el cierre de la heri$\mathrm{da}$, se procede a bloquear el nervio auricular mayor a nivel del plexo cervical superficial con $5 \mathrm{ml}$ de bupivacaína 0,5\% para mejorar el control de la analgesia post operatoria. Veinte minutos antes de terminar la cirugía se suspende la infusión de las drogas anestésicas (Figura 2).

El procedimiento duro $170 \mathrm{~min}$, la paciente es extubada al constatar ventilación espontánea y a los pocos minutos se despierta sin complicaciones. La paciente es transportada a la Unidad de Terapia Intensiva Pediátrica para mantener un control más estrecho de sus parámetros vitales. En ningún momento se registraron episodios de alteraciones en los patrones respiratorios como hiperpneas o apneas manteniendo una saturación de oxígeno de 99\% respirando aire ambiente.

La analgesia fue evaluada cada dos horas mediante la escala de caras de Wong-Baker en la sala de internación refiriendo puntajes de 4, 2 y 0 previo a que fuera dada de alta 6 horas después de finalizada la cirugía.

\section{Discusión}

El síndrome de Joubert es un trastorno de herencia autosómica recesiva que se caracteriza por presentar hipotonía, alteraciones en el desarrollo cognitivo, anomalías oculomotoras, quistes renales con deterioro de su función y un patrón respiratorio anómalo con episodios de apnea/hiperpnea.

Las principales características anatómicas de estos pacientes de interés para los anestesiólogos son: cabeza alargada, malformaciones del paladar, laringomalacia, micrognatia y lengua prominente.

El diagnóstico es eminentemente clínico y se confirma al detectar displasia o agenesia del vermis cerebeloso en la resonancia magnética, configurando lo que se conoce como el "signo del molar"[1] (Figura 3).

Si bien los patrones respiratorios anormales suelen desaparecer con la edad, la falta de estos no excluye la posibilidad de que puedan generarse por el estrés quirúrgico[2].

Aunque la bibliografía respalda fuertemente que la gravedad de estos patrones respiratorios disminuye con la edad, las alteraciones de las estructuras anatómicas del tallo cerebral persisten en los adultos, lo que

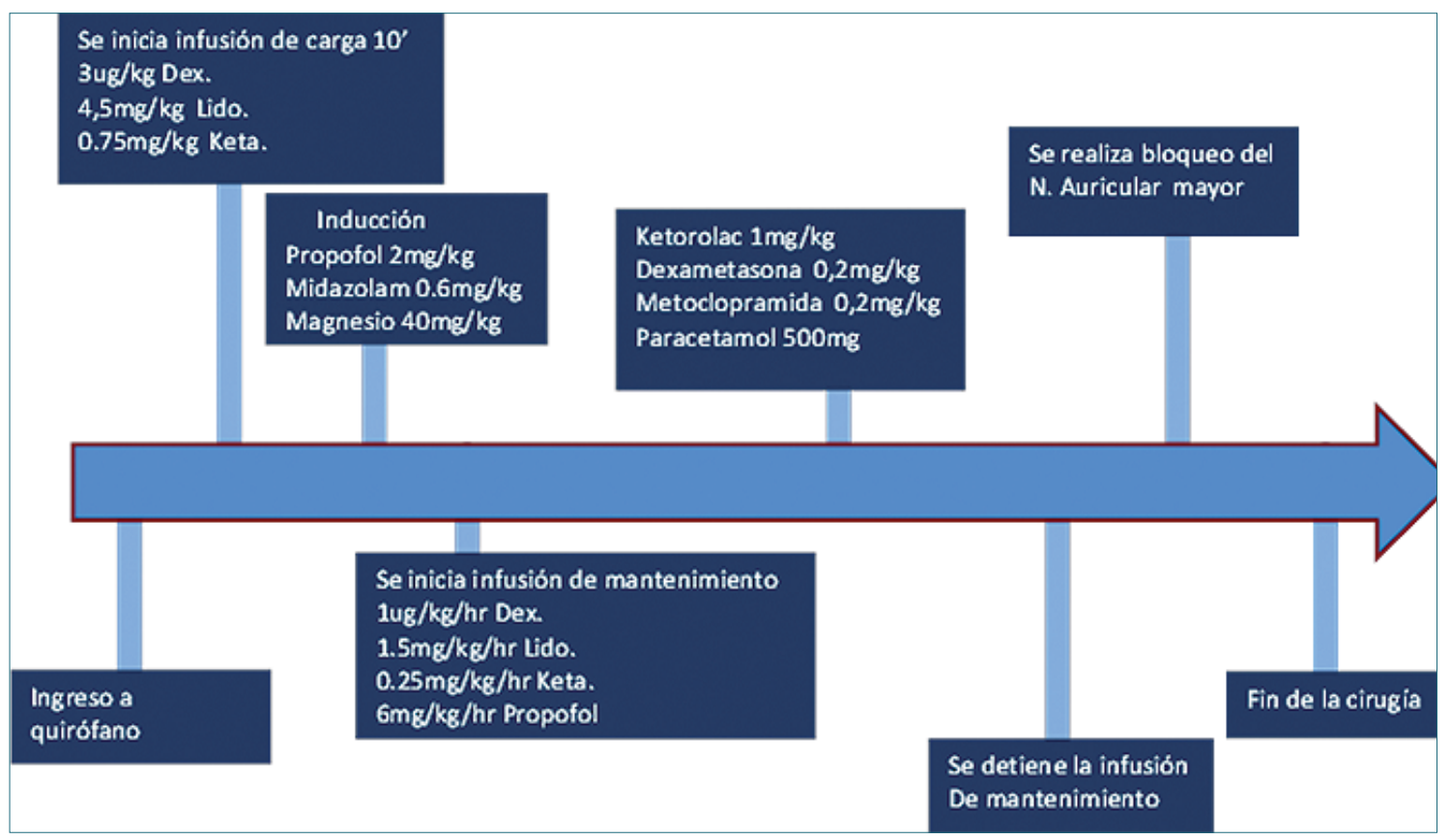

Figura 2. Línea de tiempo intraoperatoria. 


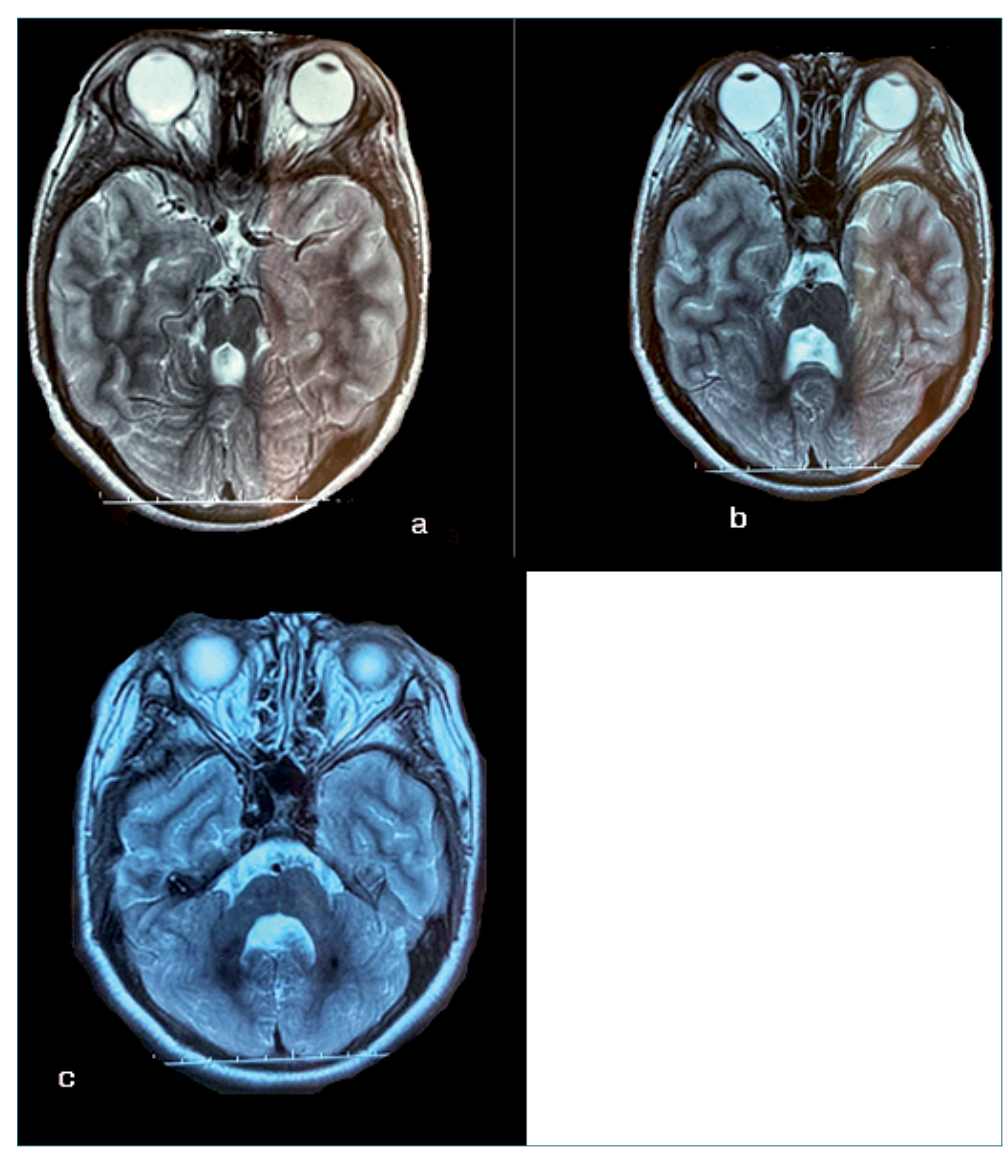

Figura 3. Imagen en T2 en la que se observa un tallo cerebral anatómicamente anormal, hipoplasia del vermis cerebeloso y dilatación del $4^{\text {to }}$ ventrículo. podría posiblemente favorecer la aparición de episodios de hiperpnea/apnea. Nosotros encontramos un reporte de caso de un paciente de 19 años quien presentó alteraciones respiratorias en el postoperatorio inmediato a pesar de su edad[2].

Reportes anteriores han descripto anestesia total intravenosa con propofol-remifentanilo[3], anestesia inhalatoria con halotano y óxido nitroso para cirugías odontológicas[4], inducción con sevofluorano y mantenimiento con Isofluorano en hernioplastia[5], el uso de dexmedetomidina-remifentanilo en cirugía cardiaca[6],[7] y la utilización de rocuronio-sugammadex para el manejo de la relajación neuromuscular.

Consideramos que este es el $1{ }^{\text {er }}$ reporte de caso publicado de una anestesia general totalmente endovenosa $100 \%$ libre de opioides sin relajación neuromuscular en un paciente con esta rara enfermedad.

El uso generalizado de opioides en anestesia data de la década de 1960.

Primero con grandes dosis de morfina, luego con el reemplazo de esta por fentanilo y, más adelante, con la aparición del remifentanilo.

A partir de 1993, se comienza a plantear la anestesia multimodal como técnica "ahorradora" de opioides bajo el concepto de que combinando fármacos con diferentes mecanismos y sitios de acción se puede lograr optimizar la analgesia y disminuir la aparición de efectos adversos.

Es así como surge el concepto de anestesia libre de opioides, suponiendo que si el uso de fármacos adyuvantes reduce el requerimiento de opioides antes, durante y después de la cirugía, entonces la combinación de muchos de estos podría posibilitar suprimir el uso de los mismos durante todo el período perioperatorio.

El interés por lograrlo radica en la extensa lista de efectos adversos que presentan estos fármacos, desde los clásicos como náuseas y vómitos, íleo y depresión respiratoria, hasta otros menos tenidos en cuenta como la inmunosupresión o la hiperalgesia.

Una de las poblaciones que más podrían beneficiarse del uso de esta técnica es justamente la de los 
pacientes con elevado riesgo de apnea postoperatoria como es el caso de aquellos con JS.

Reportamos que, en estos pacientes, la anestesia general puede ser una opción tan segura como la anestesia regional, siempre y cuando, se evite el uso de anestésicos inhalatorios y de opioides.

Asimismo, demostramos que el confort del paciente con esta técnica fue óptima durante todo el periodo transoperatorio.

\section{Conclusión}

El síndrome de Joubert es una rara enfermedad cuyas alteraciones respiratorias y de la anatomía de la vía aérea, sumada a una documentada hipersensibilidad a los opioides lo vuelve de particular interés para los anestesiólogos. La técnica anestésica total intravenosa es una estrategia muy útil para evitar también el uso de anestésicos volátiles. La dexmedetomidina combinada con lidocaina, propofol y ketamina ha demostrado ser muy útil ya que minimizan las posibilidades de trastornos respiratorios durante el postoperatorio y generan una muy buena analgesia multimodal.

\section{Conflictos de interés}

No se declara ningún conflicto de interés.

\section{Referencias}

1. Joubert M, Eisenring J-J, Robb $J P$, et al. Familial agenesis of the cerebelar vermis. A síndrome of episodic hyperpnea, abnormal eye movements, ataxia, and retardation. Neurology 19;1969:813-815 https://doi. org/10.1212MNL.19.9.813

2. Platis, C. M., Kachko, L., Trabikin, E., Simhi, E., Bahar, M. and Katz, J. Postoperative respiratory complications in Joubert syndrome. Pediatric Anesthesia, 2006; 16: 799-800. https://doi.org/10.1111/j.14609592.2006.01864.x
3. Buntenbroich, S. and Dullenkopf,

A. Total intravenous anesthesia in a patient with JoubertBoltshauser syndrome. Paediatr Anaesth, 2013; 23: 204-205. https://doi.org/10.1111/ pan.12087

4. Habre, W., Sims, C. and D'Souza, M. Anaesthetic management of children with Joubert syndrome. Pediatric Anesthesia, 1997; 7: 251-253. https://doi. org/10.1046/j.1460-9592.1997. d01-65.x

5. Vodopich, D. J. and Gordon, G. J. Anesthetic management in Joubert syndrome. Pediatric Anesthesia, 2004, 14: 871-873 https://doi.org/10.1111/j.14609592.2004.01286.x

6. Bhaskar P, John J, Sivamurthy SK et al. Anesthetic management of an infant with Joubert síndrome for cardiac surgery. J Clin Anesth 2013; 25: 488-490 https://doi.org/10.1016/j.jclinane.2013.02.013

7. Sriganesh, K., Vinay, B., Jena, S. , Sudhir, V., Saini, J. and Umamaheswara Rao, G. S. (), Anesthetic management of patients with Joubert syndrome: a retrospective analysis of a single-institutional case series. Paediatr Anaesth 2014; 24: 1180-1184 https://doi. org/10.1111/pan.12472 